\title{
Quantum spin glass: A replica-symmetric theory with positive entropy
}

\author{
Alba Theumann \\ Instituto de Física, Universidade Federal do Rio Grande do Sul, 90000 Porto Alegre, RS, Brazil
}

(Received 1 August 1984; revised manuscript recieved 17 June 1985)

\begin{abstract}
In the present paper we present a detailed analysis of quantum effects in the spin-glass transition as described by a quantum Heisenberg analogue of the Sherrington-Kirkpatrick model. The spin operators are represented in terms of two fermion fields and the problem is reduced to that of $n$ fermion levels at one site in a random time-dependent field and with an interaction delocalized in time. It is shown that within a Hartree-Fock approximation in a replica-symmetric theory one obtains a mean-field description of the transition with satisfactory zero-temperature properties. The transition is described by two order parameters: the static magnetic susceptibility and the spin-glass order parameter. The saddle-point equations obtained in this work are analogous to those of Sommers for a theory of classical spins.
\end{abstract}

\section{INTRODUCTION}

It is the purpose of this paper to present a detailed analysis of quantum effects in the spin-glass transition and to show that within a simple Hartree-Fock approximation in a replica-symmetric theory one obtains a reasonable description of the transition with satisfactory zero-temperature properties.

The spin-glass transition is described in the mean-field approximation by the model of Sherrington and Kirkpatrick $^{1}$ (SK) for classical spins, with infinite-range random exchange forces that couple all pairs of spins in the system. Current theories may be roughly classified along the following lines.

(i) Static replica theories based on the replica method ${ }^{1,2}$ to evaluate the configurationally averaged free energy. The replica-symmetric SK solution yields a negative entropy at zero temperature, and it was shown later by de Almeida and Thouless ${ }^{3}$ that this replica-symmetric solution is unstable below $T_{c}$. Since then, several theories with replica-symmetry breaking have been proposed. ${ }^{4,5}$ In particular, the solution to the model of De Dominicis and Garel $^{5}$ (DDG) yields a positive entropy although it remains unstable below $T_{c}$, and it is similar to Sommers's ${ }^{6}$ solution to the TAP equations, which we discuss below. A common feature of theories with broken replica symmetry is the occurrence of, at least, two order parameters, and the breakdown of the Fischer relation $\chi=\beta(1-q)$, for $\chi$ the static magnetic susceptibility, $q$ is the spin-glass order parameter, and $\beta=1 / k_{B} T$.

(ii) The static theory of Thouless, Anderson, and Palmer $^{7}$ (TAP) avoids the use of replicas by deriving modified mean-field equations for the random local-spin expectation values. Sommers ${ }^{6}$ obtained a solution to the configurationally averaged TAP equations in terms of two order parameters which yields a positive low-temperature entropy and also a breakdown of the Fischer relation. A detailed analysis of different static theories is given in Ref. 8.

(iii) Dynamic spin-glass theories ${ }^{9,10}$ that also avoid the use of replicas and assume a Langevin model for the spin dynamics. The spin-glass order parameters of Ref. 9 are the time-persistent terms in the average spin-correlation function and the average response function, and Sommers's static solution is recovered with certain assumptions on the dynamical behavior of the system in the thermodynamic limit.

In contrast to the enormous amount of work done on classical spin systems, quantum effects in spin glasses have been relatively slightly studied until now. ${ }^{1-14} \mathrm{Al}$ though quantum effects are expected to be irrelevant in an ordinary phase transition at finite temperature, the spinglass case may be different because time enters naturally in a quantum theory through the equation of motion of spin operators, and one may conjecture to make contact with the dynamic theories mentioned in case (iii). In fact, a natural starting point for the investigation of a quantum theory is to express the partition function as a functional integral, ${ }^{12,14}$ with the Matsubara "time" $0 \leq \tau \leq \beta$ as a new variable, and to use the replica method to obtain the averaged free energy. The resulting functional has a certain formal similarity with the dynamic generating functional for classical spins, ${ }^{9}$ except that in the quantum case one is forced to deal both with time and replicas.

In a previous paper $^{14}$ we studied a simple model obtained by setting $g=1$ in the Hamiltonian of Eq. (1). Without realizing that in this limit the model is, in fact, exactly soluble, the problem was treated in the "static" and replica-symmetric approximations as in Ref. 12. This does not invalidate the results, because the "static" approximation coincides with the exact solution, as shown in Sec. IV. We analyzed this solution also below $T_{c}$ to show explicitly that it almost reproduces the SK results, except for the occurrence of two order parameters and a breakdown of the Fischer relation as in the more accurate theories for classical spins discussed in cases (i)-(iii).

In the present paper we study the quantum Heisenberg spin glass by representing the spin operators as bilinear combinations of two fermion fields. We show that the configurational averaged free energy can be expressed as 
an effective "free energy" for localized fermions in a random time-dependent field and with a retarded two-body interaction, averaged over the Gaussian distribution of random fields. Replica symmetry is assumed throughout this work and the effective "free energy" can be written as a stationary functional of renormalized fermion propagators. In the simple Hartree-Fock approximation the saddle-point equations are analogous to those of the theories of Sommers ${ }^{6}$ and DDG. ${ }^{5}$ The solution yields a positive entropy at low temperatures, but we suspect it, without proof, of being unstable below $T_{c}$, as in the DDG model. 5

In Sec. II the general formal theory is described, the Hartree-Fock approximation is discussed in Sec. III, while a detailed discussion and comparison with other works are left for Sec. IV.

\section{GENERAL FORMULATION}

Quantum effects in spin glasses are studied $^{11,12}$ by means of the Hamiltonian

$H_{g}=-\sum_{i, j} J_{i j}\left[g \sigma_{i}^{z} \sigma_{j}^{z}+(1-g) \frac{1}{2}\left(\sigma_{i}^{+} \sigma_{j}^{-}+\sigma_{i}^{-} \sigma_{j}^{+}\right)\right]$

in the Heisenberg limit $g=\frac{1}{2}$. The spin operators satisfy the commutation rules $\left[\sigma_{i}^{+}, \sigma_{j}^{z}\right]=\mp \delta_{i j} \sigma_{i}^{ \pm},\left[\sigma_{i}^{+}, \sigma_{j}^{-}\right]$ $=2 \delta_{i j} \sigma_{i}^{z}$.

We choose to represent the spin operators by bilinear combinations of fermions with spin, localized at lattice sites $i$,

$$
\begin{aligned}
& \sigma_{i}^{z}=\frac{1}{2}\left(\psi_{i \uparrow}^{*} \psi_{i \uparrow}-\psi_{i \downarrow}^{*} \psi_{i \downarrow}\right), \\
& \sigma_{i}^{+}=\psi_{i \uparrow}^{*} \psi_{i \downarrow}=\left(\sigma_{i}^{-}\right)^{*},
\end{aligned}
$$

where the operators $\psi_{i s}^{*}, \psi_{j s}$ satisfy fermion anticommutations rules and $s=\uparrow$ or $\downarrow$. Expression of the spin operators in terms of fermions introduces a difference between the previous work ${ }^{11-13}$ and ours. While in the aforementioned papers the spin eigenstates at every site belong to the same irreducible representation $|m s\rangle$, in the present work the eigenstates are labeled by the eigenvalues $n_{i s}$ of the occupation number operator $\psi_{i s}^{*} \psi_{i s}, n_{i s}=0$ or 1 , and the eigenvalues of $\sigma_{i}^{2}$ may be $s(s+1)=\frac{3}{4}$ or zero.

As in the SK model, ${ }^{1}$ the random $J_{i j}$ are infinite ranged, with a Gaussian probability distribution of zero mean and variance $\left[J_{i j}^{2}\right]_{\mathrm{CA}}=J^{2} 4 / N, N$ being the number of sites. We indicate by $[\cdots]_{C A}$ the average over $d_{i j}$.

The grand-canonical partition function is expressed as a functional integral: ${ }^{15}$

$Z=\int \mathscr{D} \psi^{\dagger} \psi \exp \left(\sum_{j} A_{j}^{0}+\sum_{i, j} \beta J_{i j} \sum_{v} \mathbf{S}_{i}(v) \cdot \mathbf{S}_{j}(-v)\right)$,

$A_{j}^{0}=\sum_{\omega, s} \psi_{j s}^{*}(\omega) \psi_{j s}(\omega)(i \omega+\mu)$,

$S_{j}^{z}(v)=\frac{1}{2} \sum_{\omega}\left[\psi_{j \uparrow}^{*}(\omega+v) \psi_{j \uparrow}(\omega)-\psi_{j \downarrow}^{*}(\omega+v) \psi_{j \downarrow}(\omega)\right]$,

$S_{j}^{+}(v)=\sum_{\omega} \psi_{j \uparrow}^{*}(\omega+v) \psi_{j \downarrow}(\omega)=S_{j}^{x}(v)+i S_{j}^{y}(v)$,

$S_{j}^{-}(v)=\sum_{\omega} \psi_{j \downarrow}^{*}(\omega+v) \psi_{j \uparrow}(\omega)=S_{j}^{x}(v)-i S_{j}^{y}(v)$,

where $\mu$ is a chemical potential to be fixed by the condition of having, on the average, one electron per site, $\omega=(2 m+1) \pi$ and $v=2 \pi l$, are the Matsubara frequencies, $\beta=1 / k_{B} T$, and $\psi_{i s}^{*}(\omega), \psi_{i s}(\omega)$ are complex anticommuting Grassmann variables that satisf $\mathrm{y}^{16}$

$$
\begin{aligned}
& \psi_{\lambda}=\xi_{\lambda}+i \eta_{\lambda}, \quad \psi_{\lambda}^{*}=\xi_{\lambda}-i \eta_{\lambda}, \\
& \left\{\xi_{\lambda}, \xi_{\lambda^{\prime}}\right\}=\left\{\eta_{\lambda}, \eta_{\lambda^{\prime}}\right\}=\left\{\xi_{\lambda}, \eta_{\lambda^{\prime}}\right\}=0, \\
& \int d \xi_{\lambda}=\int d \eta_{\lambda}=0, \quad \int \xi_{\lambda} d \xi_{\lambda}=\int \eta_{\lambda} d \eta_{\lambda}=1 .
\end{aligned}
$$

In Eq. (8) the index $\lambda$ stands for the set $(i, s, \omega)$. Bilinear combinations of Grassmann variables are commuting entities that can be treated as $c$ numbers in Eq. (5); therefore we use the replica method ${ }^{12-14,15}$ to write the configurational averaged (CA) thermodynamic potential per site:

$$
\Omega=-\frac{1}{\beta N} \lim _{n \rightarrow 0} \frac{\left[Z^{n}\right]_{C A}-1}{n},
$$

where

$$
\left[Z^{n}\right]_{\mathrm{CA}}=\int\left(\prod_{\alpha} \mathscr{D} \psi_{\alpha}^{*} \psi_{\alpha}\right) \exp \left(\sum_{j, \alpha} A_{j \alpha}^{0}+\frac{\beta^{2} J^{2} 2}{N} \sum_{v, v^{\prime}} \sum_{\substack{\alpha, \alpha^{\prime} \\ t, t^{\prime}}} M_{\alpha \alpha^{\prime}}^{* t t^{\prime}}\left(v, v^{\prime}\right) M_{\alpha \alpha^{\prime}}^{t t^{\prime}}\left(v, v^{\prime}\right)\right)
$$

where $t=x, y, z$ and

$$
M_{\alpha \alpha^{\prime}}^{t t \prime}\left(v, v^{\prime}\right)=\sum_{i} S_{i \alpha}^{t}(v) S_{i \alpha^{\prime}}^{t^{\prime}}\left(v^{\prime}\right)=M_{\alpha \alpha^{\prime}}^{* t t^{\prime}}\left(-v,-v^{\prime}\right)
$$

Here, $\alpha=0,1, \ldots, n$ is the replica index and $A_{j \alpha}^{0}$ is the same as in Eq. (6) with the $\alpha$-dependent variables $\psi_{j s}^{\alpha *}(\omega)$, $\psi_{j s}^{\alpha}(\omega)$. The reduction to a one-site problem is achieved by the introduction of complex fields,

$$
Q_{a \alpha^{\prime}}^{t t^{\prime}}\left(v, v^{\prime}\right)=\left[Q_{a \alpha^{\prime}}^{t, t^{\prime}}\left(-v,-v^{\prime}\right)\right]^{*},
$$

in order to split the $M^{*} M$ term in Eq. (10) by Gaussian integration, with the result in the thermodynamic limit from Eq. (9), 


$$
\begin{aligned}
& \beta \Omega=\lim _{n \rightarrow 0} \frac{1}{n}\left[\frac{1}{8 \beta^{2} J^{2}} \sum_{\substack{\alpha, \alpha^{\prime} \\
t, t^{\prime}}} \sum_{v, v}\left|Q_{\alpha \alpha^{\prime}}^{t t^{\prime}}\left(v, v^{\prime}\right)\right|^{2}-\ln \Lambda\right] \\
& \Lambda=\int \mathscr{D} \psi^{*} \psi \exp \left[\sum_{\alpha} A_{\alpha}^{0}+\sum_{\substack{\alpha, \alpha^{\prime} \\
t, t^{\prime}}} \sum_{v, v^{\prime}}\left[Q_{\alpha \alpha^{\prime}}^{t t^{\prime}}\left(v, v^{\prime}\right) S_{\alpha}^{t}(-v) S_{\alpha^{\prime}}^{t^{\prime}}\left(-v^{\prime}\right)\right]\right]
\end{aligned}
$$

Here, $A_{\alpha}^{0}$ and $S_{\alpha}^{t}(v)$ refer to one site and they are given by Eqs. (4)-(7), respectively, with the site index $j$ replaced by the replica index $\alpha$. The $Q_{\alpha \alpha^{\prime}}^{t t^{\prime}}$ in Eqs. (13) and (14) satisfy the saddle-point equations:

$$
\frac{1}{4 \beta^{2} J^{2}} Q_{\alpha \alpha^{\prime}}^{t t^{\prime}}\left(v, v^{\prime}\right)=\frac{\delta}{\left[\delta Q_{\alpha \alpha^{\prime}}^{t t^{\prime}}\left(v, v^{\prime}\right)\right]^{*}} \ln \Lambda=\left\langle S_{\alpha}^{t}(v) S_{\alpha^{\prime}}^{t^{\prime}}\left(v^{\prime}\right)\right\rangle
$$

where $\langle\cdots\rangle$ in Eq. (15) indicates a thermal average with the effective single-site partition functional $\Lambda$ of Eq. (14).

A replica-symmetric, time-translational-invariant, isotropic solution to Eq. (15) is of the form

$$
\begin{aligned}
& Q_{\alpha \alpha^{\prime \prime}}^{t t^{\prime}}\left(v, v^{\prime}\right)=4 \beta^{2} J^{2}\left(\frac{\bar{\chi}(v)}{2}+q(v)\right) \delta_{v^{\prime},-\nu} \delta_{t, t^{\prime}}, \\
& Q_{\alpha \alpha^{\prime}}^{t^{\prime}}\left(v, v^{\prime}\right)=4 \beta^{2} J^{2}\left(q(v)-\frac{\bar{\chi}(v)}{2}\right) \delta_{v^{\prime},-\nu} \delta_{t, t^{\prime}} \text { for } \alpha \neq \alpha^{\prime},
\end{aligned}
$$

where $\bar{\chi}(v)$ and $q(v)$ are necessarily real parameters. It is obtained by introducing Eq. (16) in Eqs. (13)-(15),

$$
\beta \Omega=12 \beta^{2} J^{2} \sum_{v} \bar{\chi}(v) q(v)-\lim _{n \rightarrow 0}\left[\frac{1}{n} \ln \Lambda\right],
$$

where $\Lambda$ is now given by

$$
\begin{aligned}
\Lambda=\int \mathscr{D} \psi^{\dagger} \psi \exp \mid & \sum_{\alpha} A_{\alpha}^{0}+4 \beta^{2} J^{2} \sum_{v} q(v) \sum_{\alpha} \mathbf{S}_{\alpha}(v) \cdot \sum_{\alpha^{\prime}} \mathbf{S}_{\alpha^{\prime}}(-v) \\
& \left.+4 \beta^{2} J^{2} \sum_{v} \frac{\bar{\chi}(v)}{2}\left(\sum_{\alpha} \mathbf{S}_{\alpha}(v) \cdot \mathbf{S}_{\alpha}(-v)-\sum_{\substack{\alpha \neq \alpha^{\prime} \\
\alpha, \alpha^{\prime}}} \mathbf{S}_{\alpha}(v) \cdot \mathbf{S}_{\alpha^{\prime}}(-v)\right)\right] .
\end{aligned}
$$

The term affected by $q(v)$ in Eq. (18) may be split again by Gaussian integration to obtain

$$
\Lambda=\int_{-\infty}^{\infty}\left(\prod_{\nu} \frac{d \xi_{v} d \eta_{v}}{(2 \pi)^{3}}\right) \exp \left(-\frac{1}{2} \sum_{\nu}\left|\mathbf{v}_{v}\right|^{2}\right) e^{-n \tilde{\Omega} \beta},
$$

where the components of the vector $\mathbf{v}_{v}$ are

$$
\begin{aligned}
& v_{v}^{t}=\xi_{v}^{t}+i \eta_{v}^{t}=\left(v_{-v}^{t}\right)^{*} \\
& e^{-n \tilde{\Omega} \beta}=\int \mathscr{D} \psi^{\dagger} \psi \exp \left(\sum_{\alpha} A_{\alpha}+4 \beta^{2} J^{2} \sum_{v} \bar{\chi}(v) \sum_{\alpha, \alpha^{\prime}}\left(\delta_{\alpha \alpha^{\prime}}-\frac{1}{2}\right) \mathbf{S}_{\alpha}(v) \cdot \mathbf{S}_{\alpha^{\prime}}(-v)\right),
\end{aligned}
$$

and

$$
A_{\alpha}=\sum_{\omega, s}(i \omega+\mu) \psi_{s \alpha}^{*}(\omega) \psi_{s \alpha}(\omega)-2 \beta J \sum_{v}\left(2 q_{v}\right)^{1 / 2} \mathbf{v}_{v} \cdot \mathbf{S}_{\alpha}(v)
$$

What was obtained in Eq. (21) is the partition functional for a localized system of $n$ fermion levels in a time-dependent field,

$$
2 \mathbf{h}(\tau)=2 \beta J \sum_{v} \sqrt{2 q(v)} \mathbf{v} e^{i v \tau},
$$

and with a two-body interaction delocalized in time:

$$
\psi_{s_{1} \alpha}^{\dagger}(\tau) \psi_{s_{2} \alpha}(\tau) \gamma_{s_{1} s_{2}, s_{1}^{\prime} s_{2}^{\prime}}^{\alpha s^{\prime}} U\left(\tau-\tau^{\prime}\right) \psi_{s_{1}^{\prime} \alpha^{\prime}}^{\dagger}\left(\tau^{\prime}\right) \psi_{s_{2}^{\prime} \alpha^{\prime}}\left(\tau^{\prime}\right)
$$

where 


$$
\begin{aligned}
& \beta U\left(\tau-\tau^{\prime}\right)=4 \beta^{2} J^{2} \sum_{v} \bar{\chi}(v) e^{\left.i v \tau-\tau^{\prime}\right)}, \\
& \gamma_{s_{1} s_{2}, s_{1}^{\prime} s_{2}^{\prime}}^{\alpha \alpha^{\prime}}=\left(\frac{1}{2}-\delta_{\alpha \alpha^{\prime}}\right)\left[\frac{1}{4} \delta_{s_{1} s_{2}} \delta_{s_{1}^{\prime} s_{2}^{\prime}}\left(2 \delta_{s_{1} s_{1}^{\prime}}-1\right)+\frac{1}{2}\left(1-\delta_{s_{1} s_{2}}\right) \delta_{s_{1} s_{2}^{\prime}} \delta_{s_{2} s_{1}^{\prime}}\right] .
\end{aligned}
$$

The saddle-point equations give, from Eqs. (17) and (18),

$$
\begin{aligned}
& 3 \bar{\chi}(v)=\lim _{n \rightarrow 0}\left[\frac{1}{n}\left\langle\sum_{\alpha} \mathbf{S}_{\alpha}(v) \cdot \sum_{\alpha^{\prime}} \mathbf{S}_{\alpha^{\prime}}(-v)\right\rangle\right], \\
& 3 q(v)=\lim _{n \rightarrow 0}\left[\frac{1}{2 n}\left(\sum_{\alpha}\left\langle\mathbf{S}_{\alpha}(v) \cdot \mathbf{S}_{\alpha}(-v)\right\rangle-\sum_{\substack{\alpha \neq \alpha^{\prime} \\
\alpha, \alpha^{\prime}}}\left\langle\mathbf{S}_{\alpha}(v) \cdot \mathbf{S}_{\alpha^{\prime}}(-v)\right\rangle\right],\right.
\end{aligned}
$$

where $\langle\cdots\rangle$ indicates a thermodynamic average over the partition functional $\Lambda$ of Eq. (18). It is clear from Eq. (27) that $\bar{\chi}(v)$ is the configurational averaged response function to a time-dependent external field $\beta \mathrm{h}_{\mathrm{ex}} e^{i v \tau}$, and then

$$
3 \bar{\chi}(v)=\frac{\chi(v)}{\beta}
$$

for $\chi(v)$, the dynamic magnetic susceptibility.

The thermodynamic potential in Eq. (17), together with Eq. (19), has the more general expression compatible with isotropy, time translational invariance, and replica symmetry.

The results in the Hartree-Fock approximation will be discussed in the next section.

\section{APPROXIMATION OF STATIC FIELD AND INSTANTANEOUS INTERACTION}

We present in this section the evaluation of the thermodynamic potential in Eq. (17) within the simple approximation

$$
\begin{aligned}
& q(v)=q \delta_{v, 0}, \\
& \bar{\chi}(v)=\bar{\chi} \text { for all } v .
\end{aligned}
$$

The problem then reduces to a system of $n$ fermion levels at one site in the presence of a static magnetic field

$$
2 \mathbf{h}=2 \beta J \sqrt{2 q} \mathbf{v}
$$

from Eqs. (23), where $v=v_{0}$, and, with the instantaneous two-body interaction,

$$
\beta U\left(\tau-\tau^{\prime}\right)=4 \beta^{2} J^{2} \bar{\chi} \delta\left(\tau-\tau^{\prime}\right),
$$

from Eq. (25).

The action $A$ in Eq. (22) can be written within this approximation as

$$
A_{\alpha}=\sum_{\omega} \bar{\psi}_{\alpha}^{\dagger}(\omega) \underline{G}_{0}^{-1}(\omega) \bar{\psi}_{\alpha}(\omega)
$$

where

$$
\bar{\psi}_{\alpha}(\omega)=\left[\begin{array}{l}
\psi_{\uparrow \alpha}(\omega) \\
\psi_{\downarrow \alpha}(\omega)
\end{array}\right)
$$

and

$$
\underline{G}_{0}^{-1}(\omega)=\left(\begin{array}{cc}
i \omega+\mu-h_{z} & -h_{-} \\
-h_{+} & i \omega+\mu+h_{z}
\end{array}\right),
$$

with $h_{ \pm}=h_{x} \pm i h_{y}$.

The linked-cluster theorem ${ }^{17}$ allows us to write $\widetilde{\Omega}$ in Eq. (21) as

$n \widetilde{\Omega}=-n \sum_{\omega}\left\{\operatorname{Tr} \ln \underline{G}^{-1}(\omega)+\operatorname{Tr}[\underline{G}(\omega) \underline{\Sigma}(\omega)]\right\}+R$,

with

$$
\begin{aligned}
& \underline{\Sigma}(\omega)=\left(\begin{array}{cc}
\Sigma_{0}+\Sigma_{z} & \Sigma_{-} \\
\Sigma_{+} & \Sigma_{0}-\Sigma_{z}
\end{array}\right), \\
& \underline{G}^{-1}(\omega)=\underline{G}_{0}^{-1}(\omega)-\Sigma(\omega),
\end{aligned}
$$

and $R$ is the sum of all closed skeleton diagrams with the interaction of Fig. 1(a) and the full propagators $G_{s s^{\prime}}(\omega)$
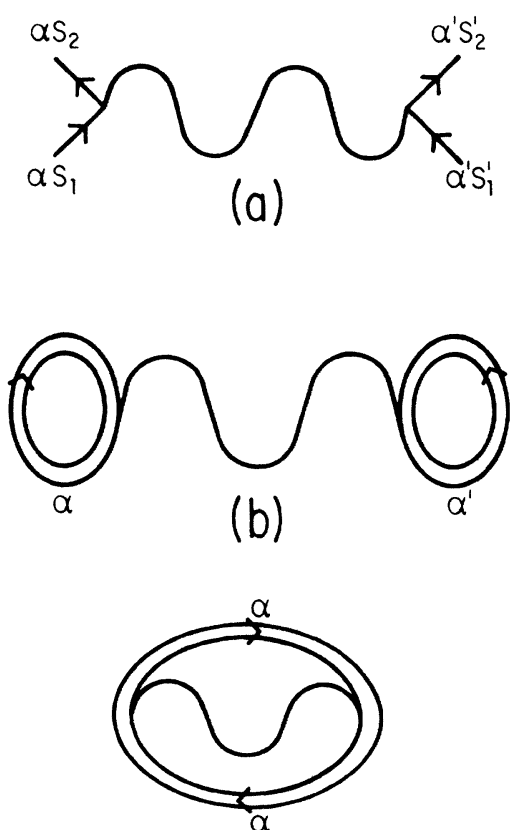

(c)

FIG. 1. (a) Retarded two-body interaction $U_{s s^{\prime}}^{\alpha \alpha^{\prime}}\left(\tau-\tau^{\prime}\right)$; (b) and (c) Hartree and exchange contribution to $\ln \phi$, respectively. Solid double lines and wavy lines indicate a dressed propagator and the interaction of (a). 
obtained by inverting the matrix in Eq. (38), with the obvious notation:

$$
\underline{G}(\omega)=\left(\begin{array}{ll}
G_{\uparrow \uparrow} & G_{\uparrow \downarrow} \\
G_{\downarrow \uparrow} & G_{\downarrow \downarrow}
\end{array}\right)
$$

The expression for $\widetilde{\Omega}$ in Eq. (36) is stationary with respect to variations of $\Sigma_{\lambda}(\omega), \lambda=0, z,+,-$, for fixed $\mu$, and self-consistent approximations for the self-energy are obtained from the stationary condition:

$$
\frac{\delta \widetilde{\Omega}}{\delta \Sigma_{\lambda}(\omega)}=0
$$

To lowest order, we approximate $R$ by the two diagrams shown in Figs. 1(b) and 1(c); then $\Sigma$ will be independent of $\omega$. We call

$$
\underline{\theta}=\sum_{\omega} \underline{G}(\omega)
$$

and by using the standard formula ${ }^{17}$

$$
\sum_{\omega} \frac{e^{i \omega \delta}}{i \omega-x}=\left(1+e^{x}\right)^{-1}
$$

we obtain, from Eqs. (37) and (38),

$\theta_{0}=\frac{1}{2}\left(\theta_{\uparrow \uparrow}+\theta_{\downarrow \downarrow}\right)=\frac{1}{2 D}\left(e^{\left(\mu-\Sigma_{0}\right)}+\cosh |\mathbf{h}+\boldsymbol{\Sigma}|\right)$,

$\boldsymbol{\theta}=\frac{\mathbf{h}+\mathbf{\Sigma}}{|\mathbf{h}+\mathbf{\Sigma}|} \frac{\sinh |\mathbf{h}+\mathbf{\Sigma}|}{2 D}$

where $\theta$ is a vector of components $\theta_{z}=\frac{1}{2}\left(\theta_{\downarrow \downarrow}-\theta_{\uparrow \uparrow}\right)$, $\theta_{-}=\theta_{\uparrow \downarrow}, \theta_{+}=\theta_{\downarrow \uparrow}$ in the notation of Eq. (39). Here,

$$
D=\cosh \left(\mu-\Sigma_{0}\right)+\cosh |\mathbf{h}+\mathbf{\Sigma}|,
$$

and for any vector $\mathbf{M}$,

$$
|\mathbf{M}|=\left(\boldsymbol{M}_{\mathbf{z}}^{2}+\boldsymbol{M}_{+} \boldsymbol{M}_{-}\right)^{1 / 2} .
$$

The direct $\left(R_{d}\right)$ and exchange $\left(R_{\mathrm{ex}}\right)$ contributions to $R$ in Figs. 1(b) and 1(c), respectively, give, in the limit $n \rightarrow 0$,

$$
\begin{aligned}
& R_{d}=-n 4 \beta^{2} J^{2} \bar{\chi}|\boldsymbol{\theta}|^{2}, \\
& R_{\mathrm{ex}}=n \beta^{2} J^{2} \bar{\chi}\left(3 \theta_{0}^{2}-|\boldsymbol{\theta}|^{2}\right),
\end{aligned}
$$

and hence from Eqs. (36), (47), and (48),

$$
\begin{aligned}
\beta \widetilde{\Omega}= & -\ln \left(1+e^{2\left(\mu-\Sigma_{0}\right)}+2 e^{\left(\mu-\Sigma_{0}\right)} \cosh |\mathbf{h}+\Sigma|\right) \\
& -2 \Sigma_{0} \theta_{0}+2 \Sigma \cdot \boldsymbol{\theta}+\beta^{2} J^{2} \bar{\chi}\left(3 \theta_{0}^{2}-5|\boldsymbol{\theta}|^{2}\right) .
\end{aligned}
$$

The stationarity condition in Eq. (40) yields the selfconsistent equations

$$
\begin{aligned}
& \Sigma_{0}=3 \beta^{2} J^{2} \bar{\chi} \theta_{0}, \\
& \Sigma=5 \beta^{2} J^{2} \bar{\chi} \theta .
\end{aligned}
$$

At the stationarity point, $\widetilde{\Omega}$ is given by

$$
\begin{aligned}
\beta \widetilde{\Omega}= & -\ln \left(1+e^{2\left(\mu-\Sigma_{0}\right)}+2 e^{\left(\mu-\Sigma_{0}\right)} \cosh |\mathbf{h}+\boldsymbol{\Sigma}|\right) \\
& -\frac{1}{3 \beta^{2} J^{2} \bar{\chi}} \Sigma_{0}^{2}+\frac{1}{5 \beta^{2} J^{2} \bar{\chi}}|\Sigma|^{2} .
\end{aligned}
$$

What was obtained in Eq. (52) is the effective thermodynamic potential, per level, for the system of $n$ fermion levels. $\widetilde{\Omega}$ is an explicit function of $\mu$ that satisfies, for fixed $\mu$ and from Eq. (43),

$$
\frac{\partial \widetilde{\Omega}}{\partial \mu}=-2 \theta_{0}
$$

and Eq. (53) defines $\theta_{0}$ (or $\Sigma_{0}$ ) as a function of $\mu$. The free energy should be stationary functional of $\mu$ and satisfy, for fixed $\Sigma_{0}$ (or $\theta_{0}$ ),

$$
\frac{\partial \widetilde{F}}{\partial \mu}=0, \quad \frac{\partial \widetilde{F}}{\partial \theta_{0}}=2 \mu
$$

We want to keep the average particle number per site equal to unity, that is

$$
\theta_{\uparrow \uparrow}+\theta_{\downarrow \downarrow}=2 \theta_{0}=1 \text {, }
$$

and this introduces a further constraint because Eq. (55) implies a functional relationship: ${ }^{17}$

$$
\mu=\Sigma_{0}(\mu) \text {. }
$$

The free energy is then defined through a Legendre transformation and a Lagrange multiplier:

$$
\beta \widetilde{F}=\beta \widetilde{\Omega}+2 \theta_{\alpha} \mu-\lambda \theta_{0}^{2},
$$

where $\lambda$ is determined through

$$
\frac{d \widetilde{F}}{d \mu}=0
$$

and the constraint $\theta_{0}^{2}=\frac{1}{4}$.

Equation (58) gives

$$
\lambda=\mu / \theta_{0},
$$

and we obtain, from Eq. (57),

$$
\beta \widetilde{F}=\beta \widetilde{\Omega}+\theta_{\alpha} \mu
$$

Equation (60), together with Eqs. (50), (52), and (56), give, for the free energy,

$$
\beta \widetilde{F}=-\ln (2+2 \cosh |\mathbf{h}+\mathbf{\Sigma}|)+\frac{1}{5 \beta^{2} J^{2} \bar{\chi}}|\mathbf{\Sigma}|^{2},
$$

where $\Sigma$ satisfies Eqs. (51) and (44). A solution is of the form

$$
\mathbf{\Sigma}=\frac{\mathbf{h}}{|\mathbf{h}|} \eta
$$

and then all the vectors are collinear and $\eta$ is the solution of the equation

$$
\eta=\frac{5}{2} \beta^{2} J^{2} \bar{\chi} \tanh \left(\frac{\beta J \sqrt{2 q} v+\eta}{2}\right),
$$

with $v=|\mathbf{v}|$ from Eq. (31).

Introducing Eq. (61) in Eq. (19), we obtain, for the system free energy in Eq. (17), 


$$
\beta F=12 \beta^{2} J^{2} \bar{\chi} q-\sqrt{2 / \pi} \int_{0}^{\infty} d v v^{2} e^{-v^{2} / 2}\left\{2 \ln \left[2 \cosh \left(\frac{\beta J \sqrt{2 q} v+\eta}{2}\right]\right)-\frac{\eta^{2}}{5 \beta^{2} J^{2} \bar{\chi}}\right\}
$$

where we used Eq. (30), and $\eta$ is the solution of Eq. (63).

The saddle-point equations give, for $q$ and $\bar{\chi}$,

$$
\begin{aligned}
& 12 \bar{\chi}=\int_{0}^{\infty} 2 \frac{d v}{\sqrt{2 \pi}} e^{-v^{2} / 2} \frac{v^{3}}{\beta J \sqrt{2 q}} \tanh \left(\frac{\beta J \sqrt{2 q} v+\eta}{2}\right), \\
& 12 q=\int_{0}^{\infty} 2 \frac{d v}{\sqrt{2 \pi}} e^{-v^{2} / 2}\left(\frac{\eta}{\beta^{2} J^{2} \bar{\chi}}\right)^{2} \frac{v^{2}}{5}
\end{aligned}
$$

Equations (64) - (66), together with Eq. (63), are similar to those obtained previously by Sommers ${ }^{6}$ and De Dominicis and Garel $^{5}$ for spin-glass theories of classical spins. The solutions are as follows

(i) $\underline{T \cong 0}$. To leading order we obtain

$$
\begin{aligned}
& \bar{\chi}=\frac{1}{6}\left(\pi \beta^{2} J^{2} q\right)^{-1 / 2}, \\
& 12 q=\frac{5}{4}-\frac{16 \sqrt{3}}{\sqrt{5 \pi} \beta J} e^{-\beta J \sqrt{5} / \sqrt{3 \pi}}, \\
& F=-\frac{J \sqrt{5}}{\sqrt{\pi 3}}-\frac{8 \sqrt{3}}{\beta^{2} J \sqrt{\pi 5}} e^{-\beta J \sqrt{5} / \sqrt{3 \pi}}, \\
& S=\frac{8 k}{\pi} e^{-\beta J \sqrt{5} / \sqrt{3 \pi}} .
\end{aligned}
$$

(ii) $T \approx T_{c}$. According to our interpretation of

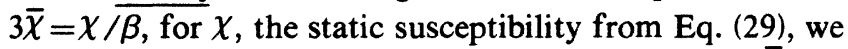
look for solutions of Eqs. (63), (65), and (66) with $\bar{\chi}>0$. To this effect we expand $\eta$ in power series of $\sqrt{q}$ on both sides of Eq. (63) to obtain

$$
\begin{aligned}
\frac{2}{5 \beta^{2} J^{2} \bar{\chi}} \eta= & \frac{1}{2} \frac{1}{1-\frac{5}{4} \beta^{2} J^{2} \bar{\chi}} \beta J \sqrt{2 q} v \\
& -\frac{1}{24} \frac{1}{\left(1-\frac{5}{4} \beta^{2} J^{2} \bar{\chi}\right)^{4}}(\beta J \sqrt{2 q} v)^{3} .
\end{aligned}
$$

it follows from Eqs. (70), (65), and (66) that

$$
\begin{aligned}
& 12 \bar{\chi}=\frac{3}{2} \frac{1}{1-\frac{5}{4} \beta^{2} J^{2} \bar{\chi}}-\frac{5}{4} \frac{\beta^{2} J^{2}}{\left(1-\frac{5}{4} \beta^{2} J^{2} \bar{\chi}\right)} q, \\
& 12 q=\frac{15}{8} \frac{\beta^{2} J^{2}}{\left(1-\frac{5}{4} \beta^{2} J^{2} \bar{\chi}\right)^{2}} q-\frac{25}{8} \frac{\left(\beta^{2} J^{2}\right)^{2}}{\left(1-\frac{5}{4} \beta^{2} J^{2} \bar{\chi}\right)^{5}} q^{2} .
\end{aligned}
$$

The solution to the equations are, for $T>T_{c}$,

$$
q=0, \bar{\chi}=\frac{1}{10 \beta^{2} J^{2}}\left[4-\left(16-10 \beta^{2} J^{2}\right)^{1 / 2}\right],
$$

while, at $T=T_{c}$,

$$
\begin{aligned}
& \frac{5}{32}\left(\beta_{c} J\right)^{2}\left(1-\frac{5}{4} \beta_{c}^{2} J^{2} \bar{\chi}_{c}\right)^{-2}=1, \\
& \bar{\chi}_{c}=\frac{1}{\beta_{c} J \sqrt{10}},
\end{aligned}
$$

which gives $\beta_{c}=4 / J \sqrt{10}$.

For $T_{\leqslant} T_{c}$ we obtain

$$
\begin{aligned}
& 12 \bar{\chi}=\frac{3}{2} \frac{1}{1-\frac{5}{4} \beta^{2} J^{2} \bar{\chi}}-2^{5} q+O\left(q^{2}\right), \\
& \frac{(12)^{2}}{10 \beta^{2} J^{2}}=\frac{9}{4} \frac{1}{\left(1-\frac{5}{4} \beta^{2} J^{2} \bar{\chi}\right)^{2}}-6(2)^{5} q+O\left(q^{2}\right),
\end{aligned}
$$

and then

$\frac{12}{\sqrt{10} \beta J}=\frac{3}{2} \frac{1}{1-\frac{5}{4} \beta^{2} J^{2} \bar{\chi}}-2^{5} q+O\left(q^{2}\right)=12 \bar{\chi}+O\left(q^{2}\right)$

$q \approx T_{c}-T$

It is worth mentioning that the solution presented here in the critical region differs from the DDG model, ${ }^{5}$ as it was found in Ref. 5 that both order parameters vanish for $T>T_{c}$. From Eqs. (29) and (74) the susceptibility $\chi$ takes the value $3 / J \sqrt{10}$ at $T_{c}$, a result also found in Ref. 12, while from Eq. (76) we obtain that, directly below $T_{c}, \chi$ sticks to the constant value

$$
\chi=\frac{3}{J \sqrt{10}}+O\left(q^{2}\right), \quad T \leq T_{c} .
$$

A similar relation has been previously obtained in the dynamical theory of classical spins ${ }^{18}$ and in the solution of TAP equations for a classical $m$-vector model ${ }^{19}$ with $m>1$.

\section{DISCUSSION AND COMMENTS}

We have shown that quantum effects are relevant to the spin-glass transition in the sense that they allow for a replica-symmetric mean-field theory with sensible lowtemperature properties, although we are aware that the correct solution cannot be obtained within a replicasymmetric formalism. There exist other theories ${ }^{5}$ that yield a positive entropy at low temperatures and they are, nontheless, unstable below $T_{c}$. Our choice of representing the spin operators in terms of two fermion fields was dictated by personal preference and it is not essential to the results. All the equations in Sec. II can be obtained within the Feynman formalism used by Bray and Moore, ${ }^{12}$ and the approximation of Eq. (30) would lead also in that representation to the problem of evaluating the free energy $\widetilde{F}$ of $n$ spins at one site with an effective "exchange constant" $4 \beta^{2} J^{2} \bar{\chi}$, in the presence of a random static magnetic field $\beta J \sqrt{2 q} v$. A mean-field solution of this site problem reproduces our results, as shown below. What is essential to the consideration of quantum spins is the occurrence of the site "local moment" $\eta$ that follows adiabatically the random field through the mean-field equation (63), and also that the susceptibility $\chi(v)$ is an order parameter of the theory as in dynamical theories of classical spins ${ }^{18,10}$ and in the $m$-vector model spinglass ${ }^{19}$ for $m>1$. This is not an artifact of the path-integral method, as can be seen by analyzing the simple model obtained by setting $g=1$ in Eq. (1). The Hamiltonian is 
now expressed only in terms of the operators $\sigma_{i}^{2}$ that commute among themselves and can be treated as $c$ numbers. Functional integrals are not needed in this case and one obtains, for the free energy, the analogue to Eq. (17),

$\beta F^{\prime}=2 \beta^{2} J^{2}\left[\left(\bar{\chi}^{\prime}\right)^{2}+2 q^{\prime} \bar{\chi}^{\prime}\right]-\frac{1}{\sqrt{2 \pi}} \int_{-\infty}^{\infty} d v e^{-v^{2} / 2} \ln \Phi^{\prime}$,

where

$q^{\prime}=\left\langle\sigma_{\alpha}^{z} \sigma_{\beta}^{2}\right\rangle, \alpha \neq \beta$

$\bar{\chi}^{\prime}=\left\langle\left(\sigma_{\alpha}^{z}\right)^{2}\right\rangle-q^{\prime}=\lim _{n \rightarrow 0}\left[\frac{1}{n}\left\langle\left(\sum_{\alpha} \sigma_{\alpha}^{z}\right)^{2}\right\rangle\right]$,

$\Phi^{\prime}=\operatorname{Tr} \exp \left[v \beta J \sqrt{2 q^{\prime}}\left(n_{\uparrow}-n_{\downarrow}\right)+\beta^{2} J^{2} \bar{\chi}^{\prime}\left(n_{\uparrow}-n_{\downarrow}\right)^{2}\right]$,

$n_{s}=\psi_{s}^{\dagger} \psi_{s}, \quad n_{s}^{2}=n_{s}$.

The chemical potential $\mu$ vanishes in this case and the trace over fermion states in Eq. (80) gives

$$
\Phi^{\prime}=2\left[1+e^{\beta^{2} J^{2} \bar{\chi}^{\prime}} \cosh \left(\beta J \sqrt{2 q^{\prime}} v\right)\right] .
$$

The free energy in Eq. (78) together with Eq. (81) is analogous to the SK result, except that also in this case we have $\bar{\chi}^{\prime}$ as an order parameter to be determined by the saddle-point equations:

$$
\frac{\partial F^{\prime}}{\partial q^{\prime}}=0, \quad \frac{\partial F^{\prime}}{\partial \bar{\chi}^{\prime}}=0
$$

Equations (78) and (81) were previously obtained ${ }^{14}$ by using path integrals in the static approximation, and the saddle-point equations for $\bar{\chi}^{\prime}$ and $q^{\prime}$ were solved at $T_{c}$ and at low temperatures. For $T=0$ we found the SK results, while for $T<T_{c}$ we obtained the same result as in Eq. (77), aside from numerical constants. This result merits further discussion. The second order parameter $\bar{\chi}^{\prime}$ appears naturally because the $g=1$ limit in Eq. (1) does not yield the Ising model, namely $\sigma_{I s}^{z}= \pm \frac{1}{2}$. As stressed in Sec. II, this is because we are not working within an irreducible representation with $s=\frac{1}{2}$, but $\sigma_{i}^{2}$ in Eq. (2) can take the values $\pm \frac{1}{2}$ or zero. This does not invalidate the theory, as the same result is obtained from any model with $s>\frac{1}{2}$. Let us consider, for instance, the case $s=1$, then the operators $\sigma_{i}^{z}$ are represented by diagonal matrices:

$$
\left(\sigma_{i}^{z}\right)_{s=1}=\left(\begin{array}{ccc}
1 & 0 & 0 \\
0 & 0 & 0 \\
0 & 0 & -1
\end{array}\right)
$$

and $\left(\sigma_{i}^{z}\right)^{2} \neq 1$. This is the case in the classical $m$-vector model with $m>1$ considered in Ref. 19, where the result of Eq. (77) was first obtained.

In Ref. 13 Sommers studied the quantum Heisenberg spin glass within the same formalism that lead to TAP equations in Ref. 6. A detailed comparison with Ref. 13 is difficult due to the difference between both methods, but there are some results in common, such as the occurrence of the dynamical susceptibility both as an order parameter and a retarded self-interaction among local spins. In Ref. 13, however, the problem was solved in the "static" approximation $\chi(\tau)=\chi$, while we used the "instantaneous" approximation $\chi(\tau)=\chi \delta(\tau)$ [Eq. (30)]. The approximation of Eq. (30) was chosen as the simplest and more tractable beyond the full static approximation, discussed in Ref. 14 for $g=1$.

To clear the doubts about the use made in this paper of the fermion representation for spin operators, we now briefly discuss the results obtained with our theory within a more conventional Feynman formalism.

The only difference resides in going to the time representation and to replace

$$
\int \mathscr{D} \psi^{\dagger} \psi \exp \left[\int_{0}^{1} d \tau\left[\psi^{*}(\tau) \frac{\partial \psi}{\partial \tau}+\mu \psi^{*}(\tau) \psi(\tau)\right]\right]\{\cdots\}
$$

by

$$
\operatorname{Tr} T\{\cdots\},
$$

where $\{\cdots\}$ is a functional of the time-dependent spin operators. We will then obtain the same formal results as in Sec. II, and with the approximation of Eq. (30) we obtain, for $\beta \widetilde{\Omega}$ in Eq. (21),

$$
e^{-n \beta \tilde{\Omega}}=\operatorname{Tr}\left[T \exp \left(2 \mathbf{h} \cdot \int_{0}^{1} d \tau \sum_{\alpha} \mathbf{S}_{\alpha}(\tau)+4 \beta^{2} J^{2} \bar{\chi} \sum_{\alpha, \alpha^{\prime}}\left(\delta_{\alpha, \alpha^{\prime}}-\frac{1}{2}\right) \int_{0}^{1} d \tau \mathbf{S}_{\alpha}(\tau) \cdot \mathbf{S}_{\alpha^{\prime}}(\tau)\right]\right]
$$

with $h$ as given in Eq. (31). Standard mean-field theory gives, for $s=\frac{1}{2}$,

$$
\beta \widetilde{\Omega}=\frac{\eta^{2}}{4 \beta^{2} J^{2} \bar{\chi}}-\ln [2 \cosh (\beta J \sqrt{2 q} v+\eta)],
$$

where

$$
\eta=4 \beta^{2} J^{2} \bar{\chi}|\langle S\rangle|=2 \beta^{2} J^{2} \bar{\chi} \tanh (\beta J \sqrt{2 q} v+\eta)
$$

is the solution of $\partial \widetilde{\Omega} / \partial \eta=0$. Equations (84) and (85) are essentially the same as Eqs. (61) and (63).

The order parameters of Eqs. (27) and (28) have been arbitrarily chosen. If the interpretation of $\bar{\chi}(v)$ as a magnetic response function is evident, what we obtain for $q(v)$ is, from Eq. (28),

$$
\begin{aligned}
3 q(v)=\mathscr{F} & \left(\left[\mathbf{S}(\tau) \cdot \mathbf{S}\left(\tau^{\prime}\right)\right\rangle\right]_{\mathrm{CA}} \\
& \left.+\left[\langle\mathbf{S}(\tau)\rangle \cdot\left\langle\mathbf{S}\left(\tau^{\prime}\right)\right\rangle\right]_{\mathrm{CA}}\right),
\end{aligned}
$$


where the inner pairs of angular brackets indicate a "thermal average" over the time- (or temperature-) dependent functional of $\Lambda$ of Eq. (19), and it is assumed explicitly that the configurational average restores time translational invariance. The definition in Eq. (79) is slightly different in order to facilitate comparison with Ref. 14.

\section{ACKNOWLEDGMENTS}

We thank W. K. Theumann for stimulating discussions. This work was supported by the Conselho Nacional de Desenvolvimento Científico e Tecnologico (CNPq) and Financiadora de Estudos e Projetos (FINEP), Brazil.
${ }^{1}$ D. Sherrington and S. Kirkpatrick, Phys. Rev. Lett. 35, 1792 (1975).

${ }^{2}$ S. F. Edwards and P. W. Anderson, J. Phys. F 5, 965 (1975).

3J. R. L. de Almeida and D. J. Thouless, J. Phys. A 11, 983 (1978).

${ }^{4}$ G. Parisi, Phys. Rev. Lett. 43, 1574 (1979); J. Phys. A 13, 1101 (1980); 13, 1887 (1980).

${ }^{5}$ C. De Dominicis and T. Garel, J. Phys. (Paris) Lett. 40, L576 (1979).

${ }^{6}$ H. J. Sommers, Z. Phys. B 31, 301 (1978); 33, 173 (1979).

${ }^{7}$ D. J. Thouless, P. W. Anderson, and R. G. Palmer, Philos Mag. 35, 593 (1977).

${ }^{8}$ A. J. Bray and M. A. Moore, J. Phys. C 13, 419 (1980).

${ }^{9}$ H. Sompolinsky and A. Zippelius, Phys. Rev. B 25, 6860 (1982).
10J. A. Hertz, A Khurana, and M. Puoskari, Phys. Rev. B 25, 2065 (1982).

${ }^{11}$ K. H. Fisher, Phys. Rev. Lett. 34, 1438 (1975); R. A. Klemm, J. Phys. C 12, L735 (1975).

${ }^{12}$ A. J. Bray and M. A. Moore, J. Phys. C 13, L655 (1980).

${ }^{13}$ H. J. Sommers, J. Magn. Magn. Mater. 22, 267 (1981).

${ }^{14}$ A. Theumann and M. Vieira Gusmão, Phys. Lett. 105A, 311 (1984).

${ }^{15}$ S. F. Edwards, J. Phys. C 8, 1660 (1975).

16Pierre Ramond, Field Theory: A Modern Primer (Benjamin/Cummings, Reading, Mass. 1981).

17J. M. Luttinger and J. C. Ward, Phys. Rev. 118, 1417 (1960).

${ }^{18}$ H. Sompolinsky, Phys. Rev. Lett. 47, 935 (1981).

${ }^{19}$ H. J. Sommers, J. Magn. Magn. Mater. 13, 139 (1979). 